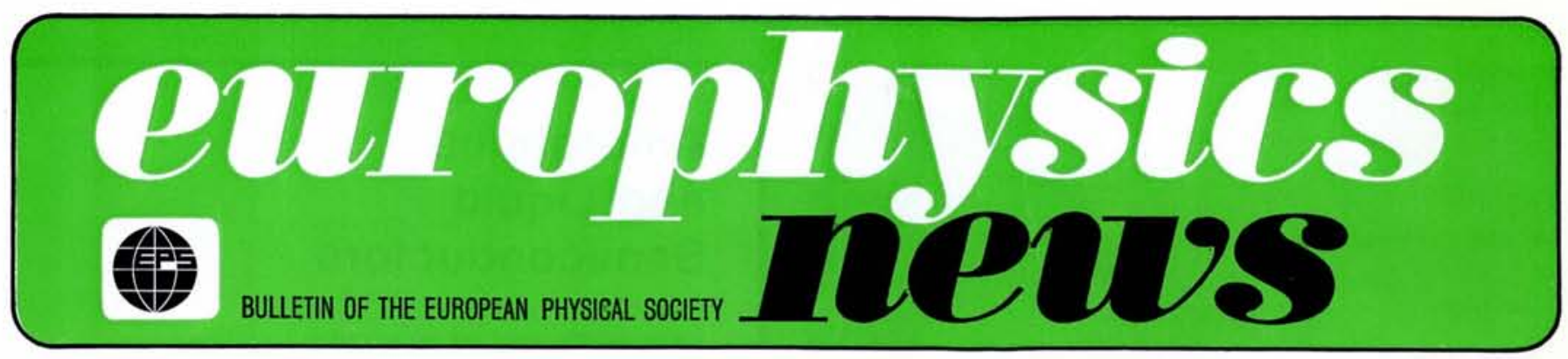

\title{
Research with High Magnetic Fields at Grenoble
}

\author{
R. Pauthenet ${ }^{\star}$ and K. Dransfeld ${ }^{\star \star}$, Grenoble \\ * Service National des Champs Intenses (C.N.R.S.) ** Hochfeld-Magnetlabor Grenoble (M.P.G.) \\ B.P. 166 F-38042 Grenoble-Cedex
}

The techniques for the production of high magnetic fields, i.e. of fields above $100 \mathrm{kG}$ ( $10 \mathrm{kG}=1$ tesla), by means of resistive coils were introduced by $\mathrm{F}$. Bitter in 1936. He constructed a water-cooled coil without iron which generated a steady field of $10 \mathrm{~T}$ inside a useful diameter of $5 \mathrm{~cm}$, consuming an electrical power of $2 \mathrm{MW}$. Towards the same period, P. Kapitza and J.D. Cockroft succeeded in producing pulsed magnetic fields of up to $40 \mathrm{~T}$ by discharging through a coil the energy stored either in a rotating generator or in a capacitor-bank.

Since 1950 several other laboratories have been equipped to produce steady fields between 15 and $22 \mathrm{~T}$ as well as pulsed fields up to $500 \mathrm{~T}$, as for example, the Clarendon Laboratory and the Royal Radar Establishment in Great-Britain, as well as the National Magnet Laboratory, the Naval Ordnance Laboratory and the Los Alamos Scientific Laboratory in the United States. We should also mention the Lebedev and the Kurchatov Institutes in the U.S.S.R., the University of Tohoku in Japan, the Low Temperature Institute in Poland, the Universities of Amsterdam in Holland and of Braunschweig in the Federal Republic of Germany, the University of Canberra in Australia, the Commissariat à l'Energie Atomique and the Centre National de la Recherche Scientifique in France.

The "Service National des Champs Intenses" (S.N.C.I.) was created in Grenoble in 1971 by the Centre National de la Recherche Scientifique (C.N.R.S.) in order to make high fields, high pressures and very low temperatures available to physicists for the study of matter under extreme conditions. Since 1972, the Max Planck Gesellschaft (M.P.G.) is associated and takes part in the further technical development and the scientific use of these installations, which we will refer to as the Grenoble Magnet Laboratory.

The laboratory has at present a total staff of 40 persons partially employed by the Centre National de la Recherche Scientifique and partially by the Max Planck Gesellschaft.

However, according to the intention of both, the C.N.R.S. and the M.P.G., all facilities and services of the Grenoble Magnet Laboratory should be available also to outside users.

The Grenoble laboratory is located close to the other C.N.R.S. Iaboratories, to the European high flux reactor (Institut Laue-Langevin) and the Centre d'Etudes Nucléaires. Grenoble has also been chosen because of its long tradition in the field of magnetism and economically for the availability of hydroelectric power.

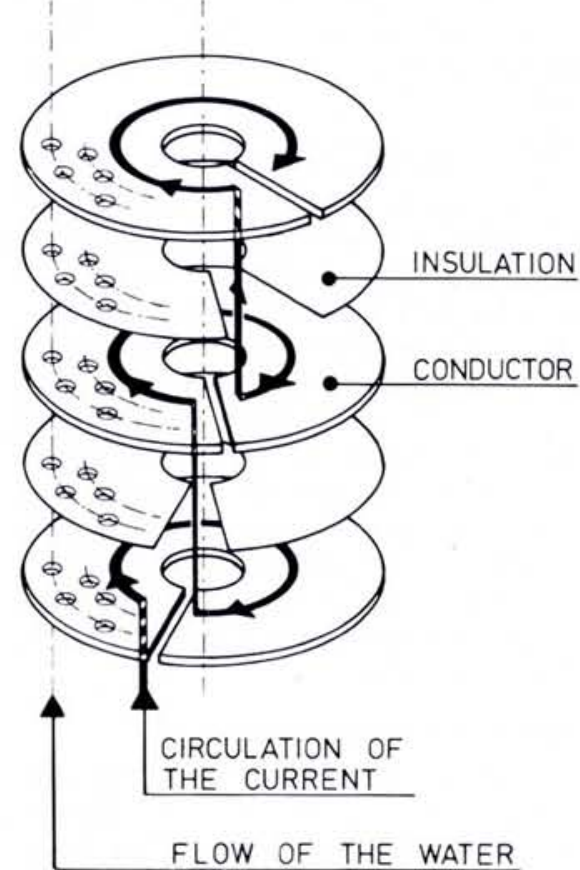

Fig. 1

The flow of current and water in a Bitter-magnet.
First we will now describe the technical facilities of the laboratory and further down give a summary of its scientific activities.

\section{The Technical Facilities}

Steady Magnetic Fields

An electrical power of $10 \mathrm{MW}$ has been installed and can be delivered via transformers. Thyristors are used for rectification and stabilisation, delivering a maximum d.c. current of $30000 \mathrm{~A}$ at $333 \mathrm{~V}$. The long-time stability of the current is better than $\pm 5 \times 10^{-5}$ and - for an interval of only 10 minutes - the current fluctuations in the frequency range from $0-10 \mathrm{kHz}$ are smaller than $5 \times 10^{-6}$ of the d.c. value.

For the water cooling of the coils, de-ionized and de-mineralized water can be pumped through the coils at a pressure of $25 \mathrm{~atm}$ at a rate of $100 \mathrm{~m}^{3} / \mathrm{h}$.

Our present 6 coils (some of them horizontal) are of the Bitter-type as schematically shown in figure 1: circular copper plates, one on top of the other, are in electrical contact only over a small part of their section, allowing the current to flow in a circular way as indicated. The holes in each disc are aligned to form a channel for the passage of the cooling water. Figure 2 shows a coil just before assembly consuming a power of $10 \mathrm{MW}$ for the production of a field of $20 \mathrm{~T}$ inside a useful diameter of $5 \mathrm{~cm}$. With a somewhat modified type of coil, a so-called polyhelix-coil, which we

\section{Contents}

Research with High Magnetic

Fields at Grenoble

Observational Paradoxes in Ex-

tragalactic Astronomy

Meetings 


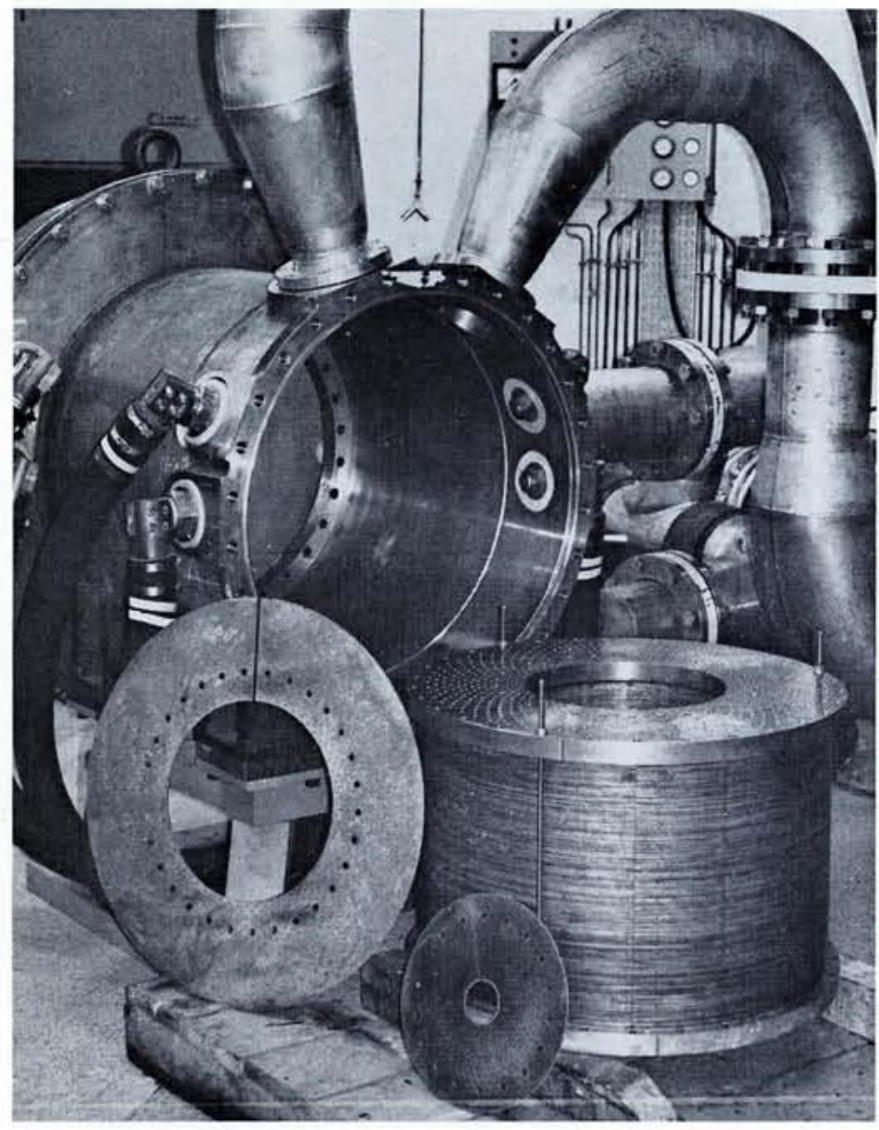

\title{
Amorphous and Liquid Semiconductors
}

\author{
Proceedings of the 5th \\ International Conference \\ held at Garmisch-Partenkirchen \\ F.R. of Germany \\ 3-8 September 1973
}

Edited by

J. Stuke University of Marburg

W. Brenig Technical University of Munich

\author{
2 volumes of 1470 pages - 183 papers \\ subject/author index \\ illustrated - cloth - $176 \times 250 \mathrm{~mm}$ \\ ISBN 0850660661 \\ $£ 30.00$ net per set of 2 volumes
}

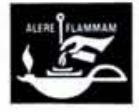

TAYLOR \& FRANCIS LTD

10-14 Macklin Street

London WC2B 5NF

Fig. 2

$10 \mathrm{MW}$ Bitter-magnet with Bitter-plates before assembly. The outside diameter of the magnet is almost one meter.

develop here in co-operation also with the Clarendon Laboratory, we expect to achieve soon fields of $22 \mathrm{~T}$ inside a bore of the same dimensions. The maximum magnetic field can be reached in two minutes, but it can be programmed to follow for example a triangular or sinusoïdal modulation.

\section{Pulsed Magnetic Fields}

In collaboration with the French Atomic Energy Commission, close to Grenoble, a laboratory has been installed for the short time production of very high fields by the "implosion technique": First a field of about $7 \mathrm{~T}$ is established in a cylindrical steel tube of $10 \mathrm{~cm}$ diameter. Subsequently this tube and the enclosed magnetic flux is radially compressed by outside explosive charges, and consequently the inside field rises to $400 \mathrm{~T}$ within $10 \mu \mathrm{sec}$ after the firing. During this time, an axially introduced sample can be observed optically under these high fields.

Transient fields of up to $120 \mathrm{~T}$ can also be achieved in a non-destructive way be passing -in a single loop around the sample- a current of $100000 \mathrm{~A}$ from a capacitor-bank.

\section{High Pressure Facilities}

Strictly hydrostatic pressures in fluid surroundings can be generated here up to $15 \mathrm{kbar}$, and quasi-hydrostatic pressures of the order of 100 kbar are being reached by means of a hydraulic press. There is a long tradition at Grenoble in the field of high pressure physics, measuring for example, the magnetization under pressure in high fields (10 T) and down low temperatures ( $2 \mathrm{~K}-300 \mathrm{~K})$, and synthesizing new materials under pressure.

\section{Low Temperatures Facilities}

The low temperature section is concerned mainly with the development of superconducting coils. At present this section has built a superconducting coil producing a field of $14 \mathrm{~T}$ of excellent homogeneity and stability. In the future, after the advent of $\mathrm{Nb}_{3} \mathrm{Sn}$ and $\mathrm{V}_{3} \mathrm{Ga}$ multifilament wires this group will seriously study the feasibility of a hybrid-magnet for Grenoble producing steady fields exceeding $25 \mathrm{~T}$. Last - not least - this group is supplying the Grenoble Magnet Laboratory with liquid helium.

\section{The Scientific Activity}

The scientific program is only partially carried out by our own staff, to a considerable degree, it has been initiated and performed by our guests, mostly from universities, but also from industrial laboratories. The total scientific program is reviewed at regular intervals by an international advisory board.

The program includes the following areas:

\section{Magnetism}

In solids, the magnetic order is defined by two principal energies. The exchange energy and the anisotropy energy. With the exchange field, one associates usually an equivalent magnetic field, the molecular field, which is proportional to the exchange integral between wave-functions of interacting atoms and to their spins; this field can range from several thousand gauss (as for some rare earth salts with Curie- or Néel-Temperatures of a few degree $\mathrm{K}$ ) up to several million gauss for substances whose ordering temperatures approaches $1000 \mathrm{~K}$ (as for example for iron and spinel ferrites). The anisotropy energy can be described by an anisotropy field, which is, in general, much smaller than the exchange field, but in certain rare earth alloys, it can also attain values of $200000 \mathrm{G}$, comparable with the exchange field.

The exchange integral depends on the distance between the magnetic atoms, and the application of pressure 
makes it possible to study this variation, by observing, for example, the pressure dependence of the ordering temperature.

Finally, it is well known that the magnetic saturation is the more perfect the lower the temperature is at which the sample is kept. The paramagnetic order depends on the ratio: (Magnetic field/Temperature). For example, for a gadolinium ion, the magnetization amounts to only $1 \%$ of its saturation value even when a field of $130 \mathrm{kG}$ is applied at liquid helium temperatures.

The fields available at the Grenoble Magnet Laboratory are sufficient for most substances to reach the exchange and anisotropy fields. The possibility to vary at the same time pressure and temperature is clearly of great use for our studies of magnetism.

A number of experiments have already been carried out in this general area, mostly by physicists from Grenoble (R. Lemaire and coworkers) but also by visitors from Paris (R. Fruchart et al.), Strasbourg (A. Meyer et al.), Toulouse (M. Carrara et al.), Nancy (M. Adam, M. Picard et al.). Here are some examples:

- The mechanism of magnetization in strongly anisotropic single crystals, both paramagnetic and ferromagnetic, and the determination of the anisotropy constants (Rareearth alloys).

- The determination of the spontaneous magnetization of materials with high magnetic anisotropy in polycristalline samples.

- Measurements of the magnetization in systems of atoms or ions weakly coupled by exchange.

$\theta \mathrm{deg} / \mathrm{cm}$
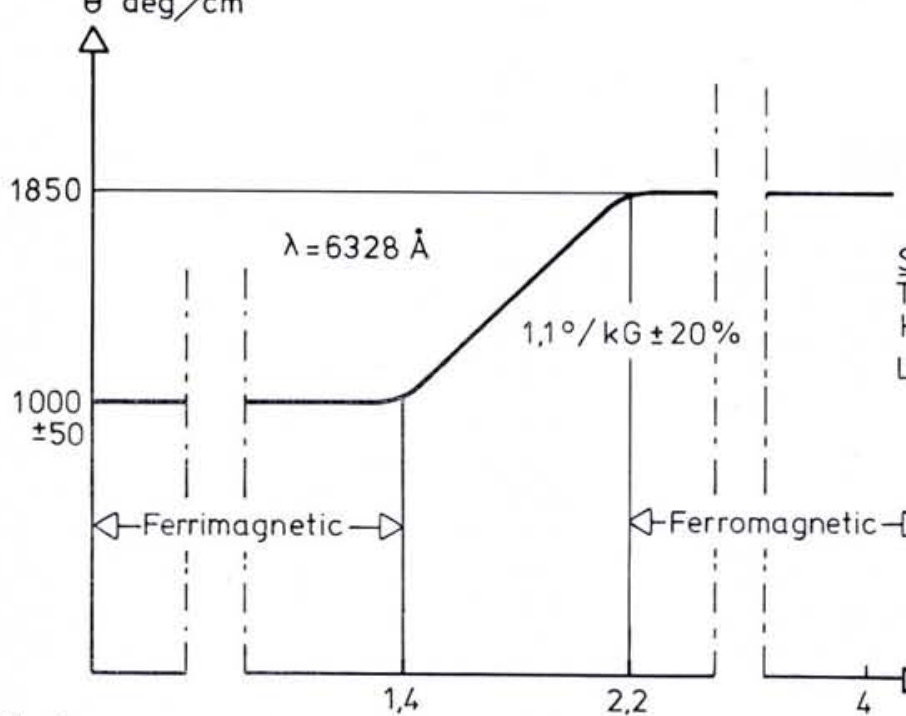

Fig. 3

A measurement of the Faraday-Rotation in Yttrium Iron Garnet at extremely high fields shows the region of a canted spin arrangement between the ferrimagnetic and ferromagnetic Phases.
- The magnetization of small ferroferri- or antiferromagnetic particles.

- The magnetization of dilute alloys (magnetic atoms in a non-magnetic matrix).

- The itinerant ferromagnetism of certain alloys, $\mathrm{Pt}-\mathrm{Ni}$ for example.

- The susceptibility of the conduction electrons in some ferromagnetic metals, from the observation of the susceptibility in strong fields.

- A large domain of investigations is the study of field induced transitions between states of different magnetic order; for example, Faraday rotation analysis on yttriumiron garnet in imploded fields (figure 3 ) indicates a transition from ferrimagnetism to a triangular arrangement, at 1,4 Million gauss, and then to a ferromagnetic order at $2,2 \mathrm{MG}$.

\section{Semiconductors}

The activity in Grenoble concentrates on three areas of semi-conductor research:

A. The magneto-optic investigation of exciton and impurity-states;

B. the study of cyclotron resonance and stimulated spin-flip by optical techniques;

C. the effect of magnetic fields on the diffusion of carriers in semi-con-

Some of these activities will be briefly described:

A. In semiconductors high magnetic fields can lead to a relatively strong variation of the wavefunction and energy of impurity states. This magnetically induced energy shift has been observed directly by photoluminescence in our laboratory. ductors.

\section{MAGNETIC BUBBLES}

\section{by E. Della Torre and}

\section{A. H. Bobeck}

1974. approx. 250 pages. approx. Dfl. 75.00 (about US\$28.90)

The book contains a comprehensive and integrated review of the theory, fabrication and device possibilities of magnetic bubbles. The stability, operating margins, field calculations, half bubbles are derived theoretically. Types of materials useable for these memory are discussed and the fabrication and processing steps are fully detailed. Finally, device applications are discussed.

\section{SOFT MODES IN FERRO-ELECTRICS AND ANTIFERRO- ELECTRICS}

\section{by R. Blinc and B. Zeks}

1974. 294 pages. Dfl. 90.00 (about US\$34.60)

The purpose of this book is to give a physical picture of the recently established connection between lattice dynamics and the occurence of both displacive and order-disorder type ferro-electric and antiferro-clectric phase transitions. It is the first monograph devoted to this rapidly expanding field of research. which is of interest to both solid state physicists and solid state chemists.

\section{THE GROWTH OF CRYSTALS FROM LIQUIDS}

\section{by J. C. Brice \\ 1973. 380 pages. Dfl. 80.00 (about US\$30.80)}

This monograph aims to give a short account of the theory and practice of the growth of crystals from the melt and from solutions including "fluxed melts". Attention is concentrated on the growth of crystals of a single phase, usually with dimensions of at least several millimetres, although methods for the production of thin layers are discussed briefly.

\section{NORTH-HOLLAND PUBLISHING CO.}

\section{P.O. Box 211}

AMSTERDAM

The Netherlands

Sole distributors for the U.S.A. and Canada:

American Elsevier Publishing

Company

52 Vanderbilt Avenue

NEW YORK. N.Y. 10017 
Under intense illumination and consequently a high density of excitons the interaction between them is known to lead, for example in Germanium, to their condensation to spherical electron-hole droplets. Their shape and size in zero magnetic field has already been seen by small angle light scattering. In a sufficiently large magnetic field, however, the electron hole plasma should not condense in spherical droplets but into the shape of long cylinders parallel to the magnetic field, i.e. to one-dimensional conductors, which we are planning to observe by small angle light scattering.

B. The behaviour of a two-dimensional electron gas in a strong magnetic field is presently being investigated in Grenoble by F. Koch (Munich) and by J.C. Thuillier et al. (Paris). The two-dimensional electron gas is produced by an inversion layer on the surface of either a silicon or a tellurium crystal. If a magnetic field is applied perpendicular to the surface the two-dimensional energy states have been observed directly by cyclotron resonance at far infrared frequencies (Koch) or by the Shubnikov-deHaas effect (Thuillier).

In close cooperation with a group from Montpellier (A.M. Joullie and C. Alibert) a new modulation technique has been developed to investigate the Landau levels by the reflection of light from a sample inside a magnetic field. Fig. 4 shows a typical spectrum of the reflected light from InSb in a field of 14.7 tesla exhibiting numerous Landau Transitions. Another group from Montpellier (J.L. Robert et al.) has also investigated the Landau levels by the Shubnikov-deHaas effect, in particular in mixed antimonides.

C. A visiting group (K. Hübner, Heidelberg) has carefully measured the diffusion coefficient of free carriers in InSb in strong magnetic fields. His observation in the strong field limit clearly demonstrates that the scattering rate for Coulomb scattering increases proportional to the magnetic field. These studies are extended to other semiconductors and higher fields.

\section{Linear Conductors}

Recently a Peierls-instability has been observed in the one-dimensional metal

$$
\mathrm{K}_{2} \mathrm{Pt}(\mathrm{CN})_{4} \cdot \mathrm{Br}_{0 \cdot 3} \cdot 3 \mathrm{H}_{2} \mathrm{O}(\mathrm{KCP})
$$

by diffuse $\mathrm{X}$-ray scattering, and at $\mathrm{T}=120 \mathrm{~K}$ a 3 -dimensional ordering between the Peierls distorsions of adjacent Pt-chains seems to occur. There has also been some speculation on the occurrence of high temperature superconductivity in one-dimensional systems.

In view of these speculations and of the fact that probably more new linear materials may become available within the next years, we are presently measuring the behaviour of $K C P$ in high magnetic fields. We are, in par-

\section{U) UNIVERSITY OF NIJMEGEN}

\section{Department of Physics}

Applications are invited for a one year position of a visiting professor, who has experience in one of the experimental research fields represented in the department: solid state physics, atomic and molecular physics, high energy physics and biophysics.

Applicants are expected to commence duties in September 1975.

Applications, including a curriculum vitae, an account of professional experience and publications, and the names of two referees should be sent to Prof. Dr. A. Dymanus, Faculty of Science, Toernooiveld, Nijmegen, Netherlands, where further information concerning the post and the department may be obtained.

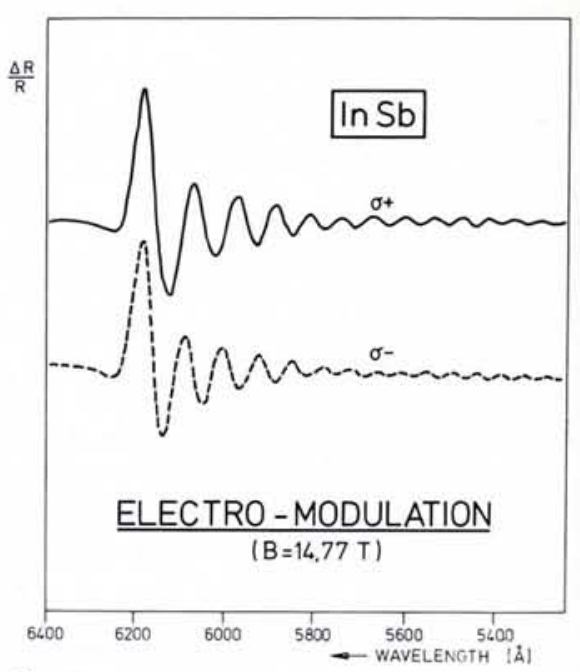

Fig. 4

The spectral distribution of the light reflected from an InSb sample in a high magnetic field.

ticular, interested in the field dependence of the transition temperatures, the corresponding fluctuations and the field induced charge transfer across the chains.

In this context it is interesting to recall a proposal by $\mathrm{H}$. Fröhlich, according ot which also 3-dimensional semimetals or semiconductors having a low enough Fermi energy should in a sufficiently high magnetic field become one-dimensional conductors, undergo a Peierls transition and exhibit possibly superconductivity.

\section{Superconducting Materials}

Together with the Grenoble Low Temperature Laboratory we are using high magnetic fields in order to better understand the nature of the transformation, which the A-15 compounds undergo above $T_{c}$, and in order to study the shape of the Fermi surface in this quasi-one-dimensional metal.

In view of the increasing importance of new high field superconducting materials not only for industrial use but also for the possible construction of a hybrid magnet here to achieve fields above 25 tesla, we are planning a cooperation with several industrial and other laboratories who are producing new materials.

The highest critical field of any superconductor - about 60 tesla for $\mathrm{Sn} \mathrm{Al}_{0.5} \mathrm{Mo}_{5} \mathrm{~S}_{6}$ - has very recently been reported by $\mathrm{O}$. Fischer et al. from the University of Geneva.

\section{Liquid Crystals}

Liquid crystals, nematic crystals for example, orient themselves already in a relatively small magnetic field. Application of strong fields influences the fluctuations of the order in the nematic phase (E. Guyon and al., Orsay) and the nematic-isotropic transition temperature (J.C. Filippini and Y. Poggi, Grenoble), which have both been studied here. 
Macromolecules, Biology and Highpolymers

As just mentioned, even moderate magnetic fields (of less than one tesla) are sufficient for the orientation of liquid crystals. For the same reason it is also possible to orient at room temperature macromolecules in a solution, if they have an anisotropic magnetic susceptibility. We are, for example, presently measuring the orientation of various biological macromolecules by recording the magnetically induced birefringence (Cotton-Mouton-Effect) and thereby getting information on their diamagnetic anisotropy.

Since not only single macromolecules but also larger biologically active particles inside a plant cell can be oriented by magnetic fields relativ to the cellmembrane, this orientation may lead to a magnetic field influence on the growth of a cell which we would like to study.

According to an experiment by $\mathrm{W}$. Haberditzl (performed at the Oxford Magnet Laboratory) also the enzymatic activity - of, for example, catalase can be strongly affected by a magnetic field. In view of the importance of this observation we are trying to confirm these results.
Let us now turn to the field of high polymers:

If magnetic fields are applied to monomers before and during the process of polymerisation the polimerized material may show a high degree of order. This question is being investigated here by $E$. Perplies (Mainz). - Another group from Mainz (J.H. Wendorff) is studying the structure of the high polymer melt (random coil or short range order) by observing the size of the Cotton-Mouton-Effect.

Finally, it should be mentioned that the single-triplet separation of the smallest molecule i.e. of molecular hydrogen has just been redetermined spectroscopically in high magneticfields (Jost et al., Grenoble).

\section{Medical Applications}

In close cooperation with the medical departments of Freiburg and Heidelberg our laboratory is using magnetic fields for the guidance of plastic catheters carrying a ferromagnetic head along the arteries of the human body, as first proposed by Montgomery from the National Magnet Laboratory, U.S.A. By means of magnetic fields it is also possible to move a magnetic fluid, used as X-ray contrast or for therapy, to a well defined place inside the body. For both these applications, it is important to develop magnets suitable for clinical use and X-ray image converters which are not affected by the relatively strong magnetic fields.

\section{Concluding Remarks}

After describing the facilities and the present scientific program we would like to point out in this Europhysics publication that the Grenoble Magnet Laboratory can be used by scientists from all countries. The formalities for outside users are simple: All what is needed is to describe the proposed research project briefly (on a form available on request) and address the application to one of us. It will be answered promptly and if the experiment can be performed in Grenoble, the laboratory will supply all its services charging only electricity and liquid helium if the consumption exceeds a certain limit. Information about the equipment here is available on request.

The number of outside guests has, in fact, steadily increased during the last two years and the quality of the present program is - to a considerable degree - due to our visitors. Last not least, we are grateful to our collaborators here for their important contribution to the operation of this laboratory.

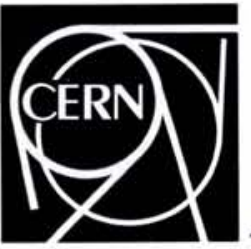

\section{European Organization for Nuclear Research}

\section{Organisation Européenne pour la Recherche Nucléaire}

CERN is a modern research laboratory, situated near Geneva, which offers first-class social and financial conditions of employment, and the opportunity to work in an international atmosphere.

\section{A junior staff position in the Theorical Study Division}

is open to scientists working on theoretical aspects of elementary particle physics or intermediate energy nuclear physics. Applicants should have a few years of post-doctoral experience and have accomplished interesting original research.

This position is for a period of three years, with the possibility of renewal for a further three years, and implies a basic salary of between 44,000 Sw.Frs., and 55,000 Sw.Frs., per annum, tax free, according to the qualifications of the selected person.
Candidates are invited to apply irrespective of nationality. However, among candidates with similar qualifications, preference will be given to nationals of CERN Member States.

Applications, including curriculum vitae and list of publications, are invited. They should be adressed to:

Professor D. Amati, Leader of the Theoretical Study Division, CERN 1211 Geneva 23, Switzerland, and received before 15 August, 1974. 